\title{
On the exit distribution of partially reflected brownian motion in planar domains
}

\author{
Athanasios BATAKIS and Viet Hung NGUYEN
}

March 21, 2022

\begin{abstract}
We show that the dimension of the exit distribution of planar partially reflected Brownian motion can be arbitrarily close to 2.
\end{abstract}

\section{Introduction}

Let $\Omega$ be a domain in $\mathbb{R}^{2}$. It is well known (see [Mak85, [JW88]) that the exit distribution of Brownian motion in $\Omega$ is carried by a borel subset of the boundary of dimension at most 1 (equal to one for simply connected domains). We are interested in the minimal dimension of sets carrying the exit distribution of partially reflected Brownian motion.

The problem is posed as follows. Consider an $(\epsilon, \delta)$ domain $\Omega$, take $F \subset \Omega$ a closed subset of the boundary of $\Omega$ and consider Brownian Motion inside $\Omega$ absorbed by $F$ and reflected on $\partial \Omega \backslash F$ (for definitions of the $(\epsilon, \delta)$ domains and of reflected brownian motion see section 2). Note $\mathcal{R}_{t}$ the above process and $\tau_{F}$ the (first) hitting time of $F$ by $\mathcal{R}_{t}$. In general, $\tau_{F}$ may not be finite or may be finite but of infinite expectation (see also the so called "trap domains" [BCM06]).

We prove the following theorem

Theorem 1.1 For all $\eta>0$ there exist a domain $\Omega$ (that can be taken simply connected) and $F \subset \partial \Omega$ such that $\mathbb{P}_{x}\left(\tau_{F}<\infty\right)=1$ and for all $x \in \Omega$ and for all $A \subset F$ of dimension $\operatorname{dim} A<2-\eta$ we have,

$$
\mathbb{P}_{x}\left(\mathcal{R}_{\tau_{F}} \in A\right)=0
$$

In particular this answers a question of B. Sapoval concerning Brownian motion as we will point out at the end of the paper: Consider a domain $\Omega$ and let $A$ be a subset of the boundary of (standard) harmonic measure equal to 1. If we change $A$ into reflecting boundary, is the dimension of the exit distribution for this new diffusion still less than 1 ?

Acknowledgement: The author would like to thank A. Ancona, L. Veron and M. Zinsmeister for many discussions that helped to clarify the original arguments and simplify the early proofs. 


\section{Definitions of the main objects}

The following definition is due to P. Jones JJon81.

Definition 2.1 We say that a (not necessarily simply connected) domain $\Omega$ is an $(\epsilon, \delta)$ domain or locally uniform if there exist constants $\epsilon$ and $\delta$ such that for all $x, y \in \Omega$ with $|x-y|<\delta$ there is a (rectifiable) curve $\gamma$ joining $x$ and $y$ satisfying

1. $\epsilon \ell(\gamma) \leq|x-y|$

2. $\epsilon \min \{|x-z|,|y-z|\} \leq \operatorname{dist}(z, \partial \Omega)$

The $(\epsilon, \delta)$-domains satisfy the so called $W^{1,2}$ - extension property, cf [Jon81]: if we note $W^{1,2}(\Omega)=\left\{f \in L^{2}(\Omega) ; \nabla f \in L^{2}(\Omega)\right\}$ with the usual Sobolev norm $\|f\|_{1,2}=\|f\|_{2}+\|\nabla f\|_{2}$, we assume that there is a bounded linear operator $T: W^{1,2}(\Omega) \rightarrow W^{1,2}(\mathbb{R})$ extending the identity of $W^{1,2}(\Omega)$.

For $f, g \in W^{1,2}(\Omega)$ define

$$
\mathcal{E}(f, g)=\int_{\Omega}<\nabla f, \nabla g>d x
$$

and

$$
\mathcal{E}_{1}(f, g)=\mathcal{E}(f, g)+\int_{\Omega} f g d x
$$

The Dirichlet form $\left(\mathcal{E}, W^{1,2}(\Omega)\right)$ is said to be regular on $\bar{\Omega}$ if $W^{1,2}(\Omega) \cap C(\bar{\Omega})$ is dense both in $\left(W^{1,2}(\Omega), \mathcal{E}_{1}^{\frac{1}{2}}\right)$ and in $\left(C(\bar{\Omega}),\|\cdot\|_{\infty}\right)$. Clearly, if $\Omega$ is a $(\epsilon, \delta)$-domain the Dirichlet form $\left(\mathcal{E}, W^{1,2}(\Omega)\right)$ is regular on $\bar{\Omega}$.

Following [Che93, BCR04] we can now define the "reflected" Brownian motion. If $\Omega$ in an $(\epsilon, \delta)$-domain, there is a strong Markov process $\mathcal{R}$ associated, with continuous sample paths. Furthermore, we can construct a family of distributions $\left(\mathcal{R}_{t}^{x}\right)_{t}$ for this process starting at every $x \in \bar{\Omega}$ (for further detail see also [FOT94]).

Take $F$ a closed subset of $\partial \Omega$ and consider $\tau_{F}$ the hitting time of $F$ for the process $\mathcal{R}_{t}^{x}$. Now if we suppose that $\mathbb{E}_{x}\left[\tau_{F}\right]<+\infty$ for at least one $x \in \Omega$, we get that for any $f \in C(F)$, the function

$$
u: x \mapsto \mathbb{E}_{x}\left[f\left(\mathcal{R}_{\tau_{F}}\right)\right]
$$

is bounded harmonic in $\Omega$ and takes the value $f$ at all regular points of $F$.

If we suppose that $\partial \Omega \backslash F$ is smooth then $u$ is the solution to the mixed Dirichlet-Neumann problem

$$
\left\{\begin{array}{l}
u \text { harmonic in } \Omega \\
\frac{\partial u}{\partial \eta}=0 \text { on } \partial \Omega \backslash F \\
u=f \text { on } F
\end{array},\right.
$$

where $\eta$ denotes the normal vector to the boundary $\partial \Omega$. 
Remark 2.2 We denote by $C_{F}(\bar{\Omega})$ the set of continuous functions on $\bar{\Omega}$ vanishing on $F$. Suppose that $W^{1,2}(\Omega) \cap C_{F}(\bar{\Omega})$ is dense in $\left.C_{F}(\bar{\Omega}),\|.\|_{\infty}\right)$. We can then define the stochastic process $\mathcal{R}_{t}^{F}$ associated. This process agrees with the previous one for all $(\epsilon, \delta)$-domains (see also [AB10]).

Let $\omega$. denote the harmonic measure of this diffusion, ie. for $x \in \Omega$ and $A \subset \partial \Omega$,

$$
\omega_{x}(A)=\mathbb{P}_{x}\left(\mathcal{R}_{\tau_{F}} \in A\right) .
$$

Remark that, from (1), for $A \subset \partial \Omega$ measurable, the function $x \mapsto \omega_{x}(A)$ is positive harmonic in $\Omega$, tending to 1 on $A$, to 0 on $F \backslash A$ and of nul normal derivative on $\partial \Omega \backslash F$.

In the following we keep this same notation.

\section{Preliminary lemmas and remarks}

Let $E \subset \mathbb{R}^{2}$ be any set and, for every covering $\mathcal{V}_{\delta}(E)$ of $E$ with discs of radius less than $\delta$, let $H_{\alpha}\left(\mathcal{V}_{\delta}(E)\right)=\sum_{B \in \mathcal{V}_{\delta}} \operatorname{diam}(B)^{\alpha}$. Consider

$$
\mathcal{H}_{\alpha}^{\delta}(E)=\inf _{\mathcal{V}_{\delta}(E)} H_{\alpha}\left(\mathcal{V}_{\delta}(E)\right) \text { and } \mathcal{H}_{\alpha}(E)=\lim _{\delta \rightarrow 0} \mathcal{H}_{\alpha}^{\delta}(E)
$$

Then, there exists an $\alpha_{0} \geq 0$ such that $\mathcal{H}_{\alpha}(E)=0$ for all $\alpha>\alpha_{0}$ and $\mathcal{H}_{\alpha}(E)=\infty$ for all $0 \leq \alpha<\alpha_{0}$. This $\alpha_{0}$ is denoted $\operatorname{dim}_{\mathcal{H}}(E)$, the Hausdorff dimension of $E$.

For a Borel measure $\mu$ we define the Hausdorff dimension of $\mu$ as

$$
\operatorname{dim}_{\mathcal{H}}(\mu)=\inf \left\{\operatorname{dim}_{\mathcal{H}}(E) ; \mu(E)>0\right\}
$$

In particular, let $\mu$ be the harmonic measure $\omega$. defined above. Using the fact that, for any $A \subset F, x \mapsto \omega_{x}(A)$ is harmonic we get that $\operatorname{dim}_{\mathcal{H}}\left(\omega_{x}\right)$ does not depend on the choice of $x \in \Omega$ and will be therefore denoted by $\operatorname{dim}_{\mathcal{H}}(\omega)$.

In this paper we are interested in the dimension of harmonic measure for partially reflected Brownian motion in domains in $\mathbb{R}^{2}$. Theorem 1.1 can now be reformulated in the following terms:

"for all $\eta>0$, there exists a uniform planar domain $\Omega$ and a closed set $F \subset \partial \Omega$ such that if $\omega$. is the harmonic measure for partially reflected Brownian motion (ie. reflected on $\partial \Omega \backslash F$, absorbed on $F$ ) we have $\operatorname{dim}_{\mathcal{H}}(\omega)>2-\eta$."

Clearly, $\operatorname{dim}_{\mathcal{H}}(\omega) \leq \operatorname{dim}_{\mathcal{H}}(F)$. Therefore the boundary of $\Omega$ will be of Hausdorff dimension $\geq 2-\eta$.

\subsection{Potential theoretic lemmas}

By "adapted cylinder" $\mathcal{C}$ to a graph $\Gamma$ of a Lipschitz function $f$ we understand the intersection of a vertical revolution cylinder of finite height centered on $\Gamma$ with the $\Gamma^{+}=\{(x, y) ; y>$ 


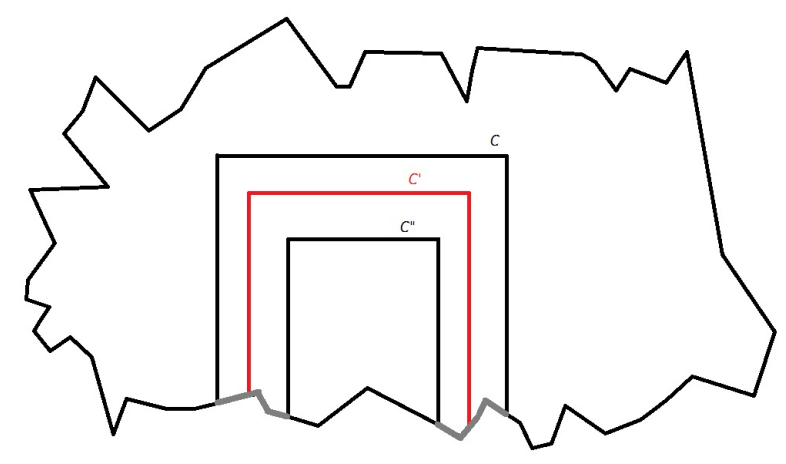

Figure 1: Boundary Harnack Principle.

$f(x)\}$. We also ask the ratio (height):(revolution radius) of $\mathcal{C}$ to be greater than 2 times $\|f\|_{L}$, la lipschitz norm of $f$.

We recall the boundary Harnack principle for reflected Brownian motion (see [BH91, [Anc90]). We say that $D$ is a Lipschitz domain if it is a Jordan domain and if the boundary is locally the graph of a Lipschitz function (with uniform lipschitz norm).

Let $D$ be a Lipschitz domain, $u$ and $v$ be positive harmonic functions on $D$ with vanishing normal derivatives on the graph between the adapted cylinder (to a graph-component of the boundary) $\mathcal{C}$ and the "sub"-adapted cylinder $\mathcal{C}$ " of the same center and revolution axis but of $\ell$ times the size, $\ell<1$ (see figure 1 ).

Proposition 3.1 If $\mathcal{C}^{\prime}$ is the "middle" cylinder of the same center and revolution axis but of $\frac{1+\ell}{2}$ times the size of $\mathcal{C}$. Then for all $x \in \partial \mathcal{C}^{\prime} \cap V$

$$
\frac{v(x)}{u(x)} \sim \frac{v(P)}{u(P)},
$$

where $P$ is the intersection point of the revolution axis of the cylinder $\mathcal{C}^{\prime}$ and of its boundary.

The multiplicative constants in the equivalence relation depend on the ratio (revolution radius):(height) of $\mathcal{C}$, on $\ell$, on the Lipschitz norm of the boundary and on the dimension of the space $n$ (here $n=2$ ) see also [Anc78].

We also need a Dirichlet-Neumann version of the maximum principle.

Proposition 3.2 Let $D$ be a planar domain, $\Gamma$ a continuous subset of the boundary of $D$, graph of a Lipschitz function, and $u$ a function harmonic in $D$ such that $\liminf _{y \rightarrow x} u(y) \geq 0$ for all $x \in \partial D \backslash \Gamma$ and $\frac{\partial u}{\partial \eta}=0$ on $\Gamma$, where $\eta$ denotes the normal vector on $\Gamma$. Then $u \geq 0$ on $D$.

This is a consequence of the unicity of solutions (see for instance [Hör94]) and the probabilistic description of these same solutions of the mixed Dirichlet-Neumann problem, described above. 


\section{$3.2 \quad$ Subsidiary results}

We will use the following result due to Benjamini, Chen and Rohde.

Theorem 3.3 (Theorem 5.1 of BCR04]) Let $\Omega$ be a locally uniform bounded planar domain. Then, $\operatorname{dim}_{\mathcal{H}}(R([0, \infty)) \cap \partial \Omega)=\operatorname{dim}_{\mathcal{H}}(\partial \Omega), \mathbb{P}_{x}$-almost surely, for all $x \in \bar{\Omega}$.

In particular, under the assumptions of the theorem, if $F \subset \Omega$ is a closed set such that $\operatorname{dim}_{\mathcal{H}}(\partial \Omega \backslash F)<\operatorname{dim}_{\mathcal{H}}(F)$ we have

$$
\mathbb{P}_{x}\left(\tau_{F}<+\infty\right)=1
$$

for all $x \in \bar{\Omega}$.

Proposition 3.4 Under the same assumptions, formula (2) implies $\mathbb{E}_{x}\left[\tau_{F}\right]<+\infty$, for all $x \in \bar{\Omega}$.

To prove this proposition we recall a result of Burdzy, Chen and Marshall.

Theorem 3.5 ([BCM06]) Let $\Omega$ be any bounded locally uniform domain and $\mathbb{B}$ a closed ball in $\Omega$. If we note $\tau_{\mathbb{B}}$ the hitting time of $\mathbb{B}$ by $\mathcal{R}_{t}$ then $\sup _{x \in \bar{\Omega}} \mathbb{E}_{x}\left[\tau_{\mathbb{B}}\right]<\infty$.

Proof of Proposition 3.4. Let $z$ be a point in $\Omega$ and $\mathbb{B}_{z} \subset \Omega$ a closed disc centered at $z$. For $x \in \Omega$ and any $s>0$,

$$
\mathbb{E}_{x}\left[\tau_{F}\right] \leq \sum_{n \in \mathbb{N}} s \mathbb{P}_{x}\left(\tau_{F} \geq n s\right) .
$$

By formula 22 for all $N \in \mathbb{N}$ there exists $s$ sufficiently big such that $\mathbb{P}_{x}\left(\tau_{F}>\frac{s}{2}\right)<\frac{1}{N}$ and $\mathbb{P}_{z}\left(\tau_{F}>\frac{s}{2}\right)<\frac{1}{N}$. Furthermore we can choose $s>2 \sup _{x \in \bar{\Omega}} \mathbb{E}_{x}\left[\tau_{\mathbb{B}_{z}}\right]$. We get that, for $n>1$,

$$
\mathbb{P}_{x}\left(\tau_{F} \geq n s\right)=\mathbb{P}_{x}\left(\tau_{F} \geq n s \mid \tau_{F} \geq(n-1) s\right) \mathbb{P}_{x}\left(\tau_{F} \geq(n-1) s\right)
$$

We can bound $\mathbb{P}_{x}\left(\tau_{F} \geq n s \mid \tau_{F} \geq(n-1) s\right) \leq \sup _{y \in \Omega} \mathbb{P}_{y}\left(\tau_{F}>s\right)$. On the other hand $\mathbb{P}_{y}\left(\tau_{F}>s\right) \leq \mathbb{P}_{y}\left(\tau_{\mathbb{B}_{z}}>s / 2\right)+\mathbb{P}_{y}\left(\tau_{\mathbb{B}_{z}}<s / 2, \mathcal{R}_{\left[\pi_{\mathbb{B}_{z}}, s\right]} \cap F=\emptyset\right)$.

Using the Markov property of $\mathcal{R}$,

$$
\mathbb{P}_{y}\left(\tau_{\mathbb{B}_{z}}<s / 2, \mathcal{R}_{\left[\tau_{\mathbb{B}_{z}}, s\right]} \cap F=\emptyset\right) \leq \mathbb{P}_{y}\left(\tau_{\mathbb{B}_{z}}<s / 2\right) \sup _{v \in \mathbb{B}_{z}} \mathbb{P}_{v}\left(\tau_{F}>s / 2\right) .
$$

Using parabolic Harnack principle (see [BCM06]) we get that there is a constant $c>1$ such that

$$
\sup _{v \in \mathbb{B}_{z}} \mathbb{P}_{v}\left(\tau_{F}>\frac{s}{2}\right) \leq c \mathbb{P}_{z}\left(\tau_{F}>\frac{s}{2}\right)<c / N
$$

We also have

$$
\mathbb{P}_{y}\left(\tau_{\mathbb{B}_{z}}<s / 2\right)<\frac{2 \sup _{x \in \bar{\Omega}} \mathbb{E}_{x}\left[\tau_{\mathbb{B}_{z}}\right]}{s}
$$

therefore, for $s$ big enough,

$$
\mathbb{P}_{x}\left(\tau_{F} \geq n s \mid \tau_{F} \geq(n-1) s\right)<1 / 2
$$

By induction we get $\mathbb{P}_{x}\left(\tau_{F} \geq n s\right) \leq\left(\frac{1}{2}\right)^{n}$ and hence $\mathbb{E}_{x}\left[\tau_{F}\right]<+\infty$. 

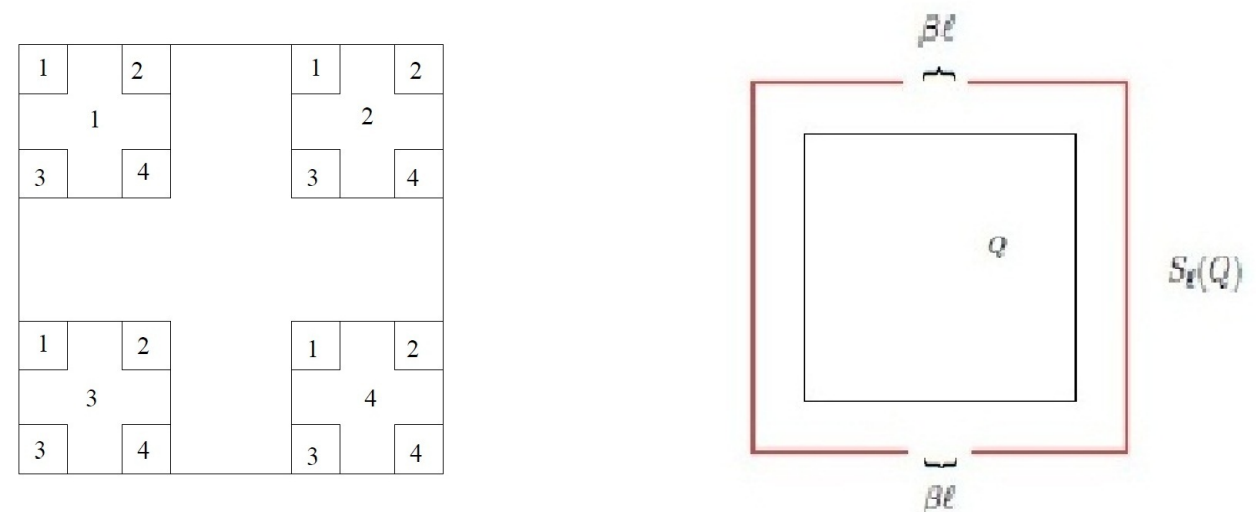

Figure 2: A. 4-corner Cantor set and its encoding. B. The squares $S_{\ell}(Q)$.

In fact we have proved that $\sup _{x \in \Omega} \mathbb{E}_{x}\left[\tau_{F}\right]<+\infty$.

\section{Proof of theorem 1.1}

Even though our proof can be carried out using only simply connected domains we have chosen to present a totally disconneted example: the constructions appear better and the lemmas get easier to write.

\subsection{Construction of the domain}

We construct, for $\alpha \in\left(0, \frac{1}{2}\right)$ a 4-corner Cantor set (fig 2,A) in the following way. We start with the square $Q=\left[-\frac{1}{2}, \frac{1}{2}\right]^{2}$ that we replace by four squares of sidelength $\alpha$ situated at the four corners of $Q$. We name these squares $Q_{1}, \ldots, Q_{4}$. We replace then each $Q_{i}, i=1, \ldots 4$ by four smaller squares of sidelength $\alpha^{2}$ situated at the corners of $Q_{i}$. We note these squares of the second generation $Q_{i j}$, where $j=1, \ldots, 4$ and so on. Let us denote $\mathbb{K}$ the Cantor set constructed in this way. We endowe $\mathbb{K}$ with the natural encoding identifying it to the abstract Cantor set $\{1, \ldots, 4\}^{\mathbb{N}}$.

Observe that $\operatorname{dim}_{\mathcal{H}}(\mathbb{K})=\left|\frac{\log 4}{\log \alpha}\right|$ and hence for $\alpha$ close to $\frac{1}{2}$ the dimension of the Cantor set is close to 2 .

The set $\mathbb{K}$ will be the absorbing part of the boundary of $\Omega$. Let us know construct the reflecting part. First of all, in order to ensure boundedness let us consider a ball $\mathbb{B}_{0}$, centered at 0 of radius, say, $10^{6}$. The domain $\Omega$ will be a subset of $\mathbb{B}_{0} \backslash \mathbb{K}$. 
Let $Q$ be a square of sidelength $\rho$ centered at $\left(x^{*}, y^{*}\right)$ and for $0<\beta<10^{-2} \rho$ and $\ell>1$ consider the "unfinished" squares

$$
S_{\beta, \ell}(Q)=\left\{(x, y) \in \mathbb{R}^{2} ;\left|x-x^{*}\right|=\left|y-y^{*}\right|=\rho \ell \text { and } x \notin\left(x^{*}-\beta \ell / 2, x^{*}+\beta \ell / 2\right)\right\}
$$

(see figure 2,B). Finally consider the blown-up version of $S_{\beta, \ell}(Q)$ (see figure 3):

$$
L_{\beta, \ell}(Q)=\left\{z \in \mathbb{R}^{2} ; \operatorname{dist}\left(z, S_{\beta, \ell}(Q)\right) \leq 10^{-6} \beta \rho\right\}
$$

Note that, if $\ell$ is less than $\frac{1}{2 \alpha}$, for any $Q$ and $Q^{\prime}$ squares of the construction of the Cantor set $L_{\beta, \ell}(Q) \cap L_{\beta, \ell}\left(Q^{\prime}\right)=\emptyset$. Consider the union of the Cantor set $\mathbb{K}$ with

$$
M_{\beta, \ell}(\mathbb{K})=\bigcup_{n \in \mathbb{N}} \bigcup_{i_{1}, \ldots i_{n}}\left(L_{\beta, \ell}\left(Q_{i_{1}, \ldots i_{n}}\right)\right)
$$

The domain $\Omega$ is defined as the complementary of this union within the ball $\mathbb{B}_{0}$ of radius $10^{6}$ :

$$
\Omega=\mathbb{B}_{0} \backslash\left(\mathbb{K} \cup M_{\beta, \ell}(\mathbb{K})\right)
$$

Remark 4.1 For $\ell>1$ and $\beta>0$ fixed the domain $\Omega$ is clearly a bounded uniform domain. Therefore we can construct partially reflected Brownian motion $\mathcal{R}_{t}$ in $\Omega$ with the partition of the boundary of $\Omega$ into an absorbing part of the boundary $F=\mathbb{K}$ and a reflecting part $\partial \Omega \backslash F$.

Note also that the Hausdorff dimension of $\partial \Omega$ equals the Hausdorff dimension of $\mathbb{K}$ if $\operatorname{dim}_{\mathcal{H}} \mathbb{K}>1$ (ie. if $\alpha>\frac{1}{4}$ ). This is because $\partial \Omega \backslash K$ consists of a countable union of rectifiable arcs and is therefore of Hausdorff dimension 1.

We will show that for $\alpha \in\left(0, \frac{1}{2}\right)$ and every $\epsilon>0$ there exists $\ell<\frac{1}{2 \alpha}$ and $\beta$ close to 0 such that the domain $\Omega$, constructed in the previous way, satisfy $\operatorname{dim}_{\mathcal{H}} \omega>(1-\epsilon) \operatorname{dim}_{\mathcal{H}} \mathbb{K}$.

\subsection{Preparatory lemmas}

Let $Q=Q_{i_{1}, \ldots, i_{n}}$ be a square of the construction of $\mathbb{K}$ of sidelength $\rho$ and let $\left(x^{*}, y^{*}\right)$ be it's center. Let $F_{\beta, \ell}(Q)=L_{\beta, \ell}(Q) \cup C_{\beta, \ell}(Q)$ where

$$
\begin{aligned}
C_{\beta, \ell}(Q)= & \left\{(x, y) ; y>y^{*}+\rho \ell \text { and }\left\|\left(x-x^{*}, y-y^{*}-\rho \ell\right)\right\|=\left(\frac{1}{2 \alpha}-\ell\right) \rho\right\} \\
& \bigcup\left\{(x, y) ; y<y^{*}-\rho \ell \text { and }\left\|\left(x-x^{*}, y-y^{*}+\rho \ell\right)\right\|=\left(\frac{1}{2 \alpha}-\ell\right) \rho\right\},
\end{aligned}
$$

see figure 3 .

Let $\tilde{D}$ be the bounded component of the complementary of $F_{\beta, \ell}(Q)$ and $D=\Omega \cap \tilde{D}$ (as in figure 3).

We consider reflected Brownian motion ${ }^{D} \mathcal{R}$ in $D$ and we note $\tau^{D}$ the hitting time of $\mathbb{K} \cup C_{\beta, \ell}(Q)$ by ${ }^{D} \mathcal{R}$. It follows on the previous discussion that for all $x \in D, \mathbb{E}_{x} \tau^{D}<\infty$ and, furthermore, $\mathbb{P}_{x}\left({ }^{D} \mathcal{R}_{\tau^{D}} \in \mathbb{K}\right)>0$. To prove this last claim one can also use the arguments of relation (8) below, applied to the domaine $D$ and to the diffusion ${ }^{D} \mathcal{R}$ respectively. 


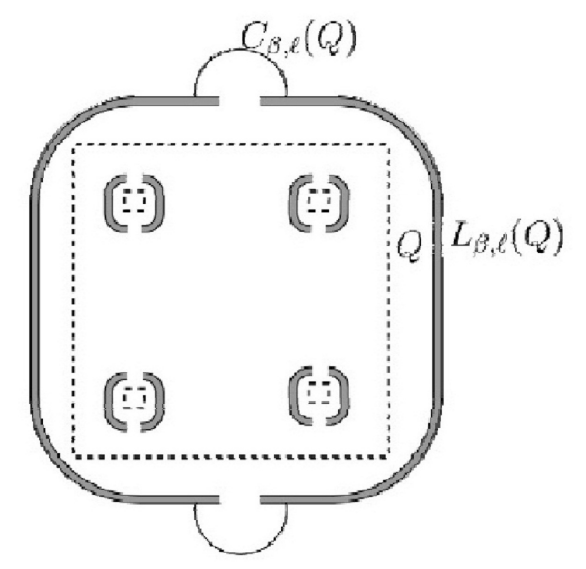

Figure 3: $F_{\beta, \ell}(Q)$

Remark 4.2 Consider $Q_{1}=Q_{i_{1}, \ldots, i_{n} a}, Q_{2}=Q_{i_{1}, \ldots, i_{n} b}$ (with $a, b=1, . ., 4$ ) two sub-cubes of $Q=Q_{i_{1}, \ldots, i_{n}}$. By symmetry we get that, if $x_{Q}=\left(x^{*}, y^{*}\right)$ is the center of $Q$,

$$
\mathbb{P}_{x_{Q}}\left({ }^{D} \mathcal{R}_{\tau^{D}} \in \mathbb{K} \cap Q_{1}\right)=\mathbb{P}_{x_{Q}}\left({ }^{D} \mathcal{R}_{\tau^{D}} \in \mathbb{K} \cap Q_{2}\right) .
$$

It follows, using Harnack's principle, that for all $\epsilon>0$ there exists $r=r_{\epsilon}>0$ (depending only on $\epsilon, \ell, \alpha$ but not on $\beta$ ) such that

$$
\left\|x-x_{Q}\right\|<r \Longrightarrow \mathbb{P}_{x_{Q}}\left({ }^{D} \mathcal{R}_{\tau^{D}} \in \mathbb{K} \cap Q_{1}\right) \leq(1+\epsilon) \mathbb{P}_{x_{Q}}\left({ }^{D} \mathcal{R}_{\tau^{D}} \in \mathbb{K} \cap Q_{2}\right)
$$

Let us now prove that for $\beta$ small enough, the harmonic functions (measures) $U_{i}()=$. $\mathbb{P} .\left({ }^{D} \mathcal{R}_{\tau^{D}} \in \mathbb{K} \cap Q_{i}\right), i=1,2$, satisfy inequality (4) in the subdomain $D^{\prime}$ of $D$ :

$$
\left.D^{\prime}=D \backslash\left(\mathbb{B}\left(\left(x^{*}, y^{*}+\rho \ell\right),\left(\frac{1}{2 \alpha}-\ell\right) \rho\right) \cup \mathbb{B}\left(\left(x^{*}, y^{*}-\rho \ell\right),\left(\frac{1}{2 \alpha}-\ell\right) \rho\right)\right) \cup \bigcup_{i=1 \ldots 4} \ell Q_{i}\right) .
$$

Remark that the closure of $D^{\prime}$ is a compact subset of $D \cup L_{\beta, \ell}(Q)$.

Lemma 4.3 For all $\epsilon>0$ there exists $\beta_{0}>0$ such that for all $\beta<\beta_{0}$ and all $x \in D^{\prime}$

$$
\mathbb{P}_{x}\left(\exists t<\tau^{D} ;{ }^{D} \mathcal{R}_{t} \in \mathbb{B}\left(x_{Q}, r_{\epsilon}\right)\right)>1-\epsilon
$$

Proof We introduce an auxilliary subdomain $D^{\prime \prime}$ of $D, D^{\prime \prime}=D \backslash \bar{B}\left(x_{Q}, r_{\epsilon}\right)$. Consider, in $D^{\prime \prime}$ the harmonic function $\zeta$ satisfying the mixed Dirichlet-Neumann boundary conditions $\zeta=0$ on $B\left(x_{Q}, r_{\epsilon}\right), \zeta=1$ on $\mathbb{K} \cup C_{b, \ell}(Q)$ and $\frac{\partial \zeta}{\partial \eta}=0$ elsewhere on $\partial D^{\prime \prime}$.

It is immediate that, since $r_{\epsilon}$ does not depend on $\beta, \zeta$ tends to 0 when $\beta$ goes to 0 . By the maximum principle 3.2 ,

$$
1-\zeta(x)<\mathbb{P}_{x}\left(\exists t<\tau^{D} ;{ }^{D} \mathcal{R}_{t} \in \mathbb{B}\left(x_{Q}, r_{\epsilon}\right)\right) .
$$

On the other hand, for every $x$ there is an $\beta_{0}$ such that $\zeta(x)<\epsilon$ for all $\beta<\beta_{0}$ and by Harnack's principle this inequality can be taken uniform in $\overline{D^{\prime}}$. 
Keeping the same notation we also have:

Lemma 4.4 Let $Q_{i}=Q_{i_{1}, \ldots, i_{n} i}, i=1, \ldots 4$, be a sub-cube of $Q=Q_{i_{1}, \ldots, i_{n}}$. Then, there $i s$ a constant $C>0$ depending only on $\alpha, \ell$ such that for all $x \in C_{\beta, \ell}\left(Q_{i}\right)$

$$
\mathbb{P}_{x}\left(\exists 0<t_{1}<t_{2}<\tau^{D} ;{ }^{D} \mathcal{R}_{t_{1}} \in B\left(x_{Q}, r_{\epsilon}\right),{ }^{D} \mathcal{R}_{t_{2}} \in C_{\beta, \ell}\left(Q_{i}\right)\right) \geq C
$$

The proof of this lemma is standard and hence omitted.

\subsection{Harmonic measure estimates}

As before, let $Q_{1}=Q_{i_{1}, \ldots, i_{n} a}, Q_{2}=Q_{i_{1}, \ldots, i_{n} b}$ be two sub-cubes of a given cube $Q=Q_{i_{1}, \ldots, i_{n}}$ of the construction of $\mathbb{K}$.

Take $U_{1}$ and $U_{2}$ to be the harmonic functions previously defined in $D$, ie. satisfying the mixed Dirichlet-Neumann boundary conditions :

$$
\left\{\begin{array} { l } 
{ U _ { 1 } = 1 \text { on } \mathbb { K } \cap Q _ { 1 } } \\
{ U _ { 1 } = 0 \text { on } ( \mathbb { K } \cap Q _ { 2 } ) \cup C _ { \beta , \ell } ( Q ) } \\
{ \frac { \partial U _ { 1 } } { \partial \eta } = 0 \text { elsewhere on } \partial D }
\end{array} \text { and } \left\{\begin{array}{l}
U_{2}=1 \text { on } \mathbb{K} \cap Q_{2} \\
U_{2}=0 \text { on }\left(\mathbb{K} \cap Q_{1}\right) \cup C_{\beta, \ell}(Q) \\
\frac{\partial U_{2}}{\partial \eta}=0 \text { elsewhere on } \partial D
\end{array}\right.\right.
$$

Thus, $U_{i}()=.\mathbb{P} .\left({ }^{D} \mathcal{R}_{\tau^{D}} \in \mathbb{K} \cap Q_{i}\right), i=1,2$.

Lemma 4.5 For every $\epsilon>0$ there existe $\beta_{0}>0$ such that for all $0<\beta<\beta_{0}$ and all $x \in D^{\prime}$,

$$
U_{1}(x)<(1+\epsilon) U_{2}(x)
$$

Proof The proof relies on lemma 4.3. By Harnack's principle there exists $C>0$ such that $U_{i}(x) \geq C U_{i}\left(x_{Q}\right)$, for all $x \in D^{\prime}$. On the other hand,

$$
\begin{aligned}
U_{i}(x) & =\mathbb{P} .\left({ }^{D} \mathcal{R}_{\tau^{D}} \in \mathbb{K} \cap Q_{i}\right)= \\
& =\mathbb{P} .\left({ }^{D} \mathcal{R}_{\tau^{D}} \in \mathbb{K} \cap Q_{i},{ }^{D} \mathcal{R}_{\left[0, \tau^{D}\right]} \cap \mathbb{B}\left(x_{Q}, r_{\epsilon}\right)=\emptyset\right)+ \\
& +\mathbb{P} .\left({ }^{D} \mathcal{R}_{\tau^{D}} \in \mathbb{K} \cap Q_{i},{ }^{D} \mathcal{R}_{\left[0, \tau^{D}\right]} \cap \mathbb{B}\left(x_{Q}, r_{\epsilon}\right) \neq \emptyset\right)
\end{aligned}
$$

Lemmas 4.3 and 4.4 imply that

$$
\mathbb{P} .\left({ }^{D} \mathcal{R}_{\tau^{D}} \in Q_{i},{ }^{D} \mathcal{R}_{\left[0, \tau^{D}\right]} \cap \mathbb{B}\left(x_{Q}, r_{\epsilon}\right)=\emptyset\right) \leq \epsilon \mathbb{P} .\left({ }^{D} \mathcal{R}_{\tau^{D}} \in Q_{i},{ }^{D} \mathcal{R}_{\left[0, \tau^{D}\right]} \cap \mathbb{B}\left(x_{Q}, r_{\epsilon}\right) \neq \emptyset\right)
$$

On the other hand, by the Markov property,

$$
\mathbb{P} .\left({ }^{D} \mathcal{R}_{\tau^{D}} \in Q_{i},{ }^{D} \mathcal{R}_{\left[0, \tau^{D}\right]} \cap \mathbb{B}\left(x_{Q}, r_{\epsilon}\right) \neq \emptyset\right) \leq \sup _{x \in \mathbb{B}\left(x_{Q}, r_{\epsilon}\right)} \mathbb{P}_{x}\left({ }^{D} \mathcal{R}_{\tau^{D}} \in Q_{i}\right)
$$

Therefore, using once more Harnack's inequality

$$
U_{i}(x) \leq(1+c \epsilon) U_{i}\left(x_{Q}\right),
$$

and the lemma's claim follows using symmetry. 
Consider now the functions $V_{1}$ and $V_{2}$ that solve the following mixed Dirichlet-Neumann problem in $\Omega$.

$$
\left\{\begin{array} { l } 
{ V _ { 1 } = 0 \text { on } \mathbb { K } \cap Q _ { 1 } } \\
{ V _ { 1 } = U _ { 1 } \text { on } \mathbb { K } \backslash Q _ { 1 } } \\
{ \frac { \partial V _ { 1 } } { \partial \eta } = \frac { \partial U _ { 1 } } { \partial \eta } \text { on } \partial \Omega \backslash \mathbb { K } }
\end{array} \text { and } \left\{\begin{array}{l}
V_{2}=0 \text { on } \mathbb{K} \cap Q_{2} \\
V_{2}=V_{1} \text { on } \mathbb{K} \backslash Q_{2} \\
\frac{\partial V_{2}}{\partial \eta}=\frac{\partial U_{2}}{\partial \eta} \text { on } \partial \Omega \backslash \mathbb{K}
\end{array}\right.\right.
$$

\subsection{Proof of theorem}

We need to show that for $\beta$ small enough $V_{i} \leq(1+\epsilon) V_{j}$, for $i, j=1,2$. Since $V_{i}(x)=$ $\mathbb{P}_{x}\left(\mathcal{R}_{\tau_{\mathbb{K}}} \in \mathbb{K} \cap Q_{i}\right)$, this inequality clearly implies that the harmonic measure for partially reflected Brownian motion $\omega$ satisfies

$$
(1+\epsilon)^{-n} 4^{-n} \leq \omega\left(Q_{i_{1}, \ldots, i_{n}}\right) \leq(1+\epsilon)^{n} 4^{-n}
$$

for all $n$ and all indices $i_{1}, \ldots i_{n} \in\{1, \ldots, 4\}$ and hence the claim.

We note

$$
C_{\beta, \ell}^{\prime}=D \cap \partial\left(\mathbb{B}\left(\left(x^{*}, y^{*}+\rho \ell\right),\left(\frac{1}{2 \alpha}-\ell\right) \rho\right) \cup \mathbb{B}\left(\left(x^{*}, y^{*}-\rho \ell\right),\left(\frac{1}{2 \alpha}-\ell\right) \rho\right)\right)
$$

For $n \in \mathbb{N}$, consider the increasing sequences of hitting times

$$
T_{n}=\inf \left\{t ; \exists t_{1}<s_{1} \ldots<t_{n-1}<s_{n-1}<t \text { s.t. } \mathcal{R}_{t_{i}} \in C_{\beta, \ell}^{\prime}, \mathcal{R}_{s_{i}} \in C_{\beta, \ell}\right\}
$$

and

$$
S_{n}=\inf \left\{s ; \exists t_{1}<s_{1} \ldots<t_{n}<s \text { s.t. } \mathcal{R}_{t_{i}} \in C_{\beta, \ell}^{\prime}, \mathcal{R}_{s_{i}} \in C_{\beta, \ell}\right\}
$$

with the convention $T_{n}=\infty\left(S_{n}=\infty\right)$ if the corresponding set is empty.

$$
\begin{aligned}
V_{i}(x) & =\mathbb{P}_{x}\left(\mathcal{R}_{\tau_{\mathbb{K}}} \in Q \cap \mathbb{K}, \mathcal{R}_{\tau_{\mathbb{K}}} \in \mathbb{K} \cap Q_{i}\right) \\
& =\sum_{n} \mathbb{P}_{x}\left(0<T_{1}<\ldots<T_{n}<\tau_{\mathbb{K}}<\infty, S_{n}=\infty, \mathcal{R}_{\tau_{\mathbb{K}}} \in \mathbb{K} \cap Q_{i}\right) \\
& =\sum_{n} \mathbb{P}_{x}\left(0<T_{1}<\ldots<T_{n}<\infty\right) \mathbb{E}_{x} \mathbb{P}_{R_{T_{n}}}\left(S_{1}=\infty, \mathcal{R}_{\tau_{\mathbb{K}}} \in \mathbb{K} \cap Q_{i}\right)
\end{aligned}
$$

where the last equality is derived by Markov's property. By lemma 4.5, for $i, j=1,2$ and all $z \in C_{\beta, \ell}^{\prime}$,

$$
\mathbb{P}_{z}\left(S_{1}=\infty, \tau_{\mathbb{K}} \in \mathbb{K} \cap Q_{i}\right) \leq(1+\epsilon) \mathbb{P}_{z}\left(S_{1}=\infty, \mathcal{R}_{\tau_{\mathbb{K}}} \in \mathbb{K} \cap Q_{j}\right)
$$

Implementing this inequality in (8) we get

$$
V_{i}(x) \leq \sum_{n} \mathbb{P}_{x}\left(0<T_{1}<\ldots<T_{n}<\infty\right) \mathbb{E}_{x} \mathbb{P}_{R_{T_{n}}}\left(S_{1}=\infty, \mathcal{R}_{\tau_{\mathbb{K}}} \in \mathbb{K} \cap Q_{j}\right)
$$

and hence $V_{i} \leq(1+\epsilon) V_{j}$, which completes the proof. 
Comments-Further Remarks: If the boundary of the domain $\Omega$ is entirely absorbing, ie. for the Laplace equation with Dirichlet boundary conditions, then harmonic measure is carried by $\partial \Omega \backslash \mathbb{K}$. This is not difficult to see. In fact, using previous notation, to get to $\mathbb{K}$ Brownian motion has to go through an infinity of conformal annuli of the type $L \beta, \ell(Q) \backslash Q$. But, at every passage of this type, there is a -bounded from below probability- to hit $L \beta, \ell(Q)$. Hence the probability that brownian motion hits $\mathbb{K}$ is 0 . Moreover, harmonic measure will be carried by a union of curves of finite length.

This answers the question of B. Sapoval mentionned in the introduction.

Another way to study the passage between Dirichlet boundary condition to the Neumann boundary condition through the mixed Dirichlet-Neumann is through a random approach. This the object of a previous work [BLZ11] that should be completed in a forthcoming artcile ABZ10.

\section{References}

[AB10] G. Allain and A. Batakis. On random processes defined through Dirichlet forms : the partially reflected Brownian motion, 2010. under redaction.

[ABZ10] A. Ancona, A. Batakis, and M. Zinsmeister. Stationary measures for brownian flights in fractal domains. Journal inconnu, 2010. under redaction.

[Anc78] A. Ancona. Principe de Harnack à la frontière et théorème de Fatou pour un opérateur elliptique dans un domaine Lipschitzien. Annales de l' Institut Fourier, Grenoble, 28 (4): 169-213, 1978.

[Anc90] A. Ancona. Théorie du potentiel sur les graphes et les variétés. In Ecole d'été de probabilités de Saint-Flour XVII, volume 1427 of Lecture Notes in Mathematics. Springer-Verlag Berlin, 1990.

[BCM06] K. Burdzy, Z-Q. Chen, and D. Marshall. Traps for reflected Brownian motion. Mathematische Zeitschrift, 252(1): 103-132, january 2006.

[BCR04] I. Benjamini, Z-Q. Chen, and S. Rohde. Boundary trace of reflecting brownian motions. Probability theory and related fields, 129(1): 1-17, 2004.

[BH91] R. F. Bass and P. Hsu. Some potential theory for reflecting brownian motion in hölder and lipschitz domains. Annals of Probability, 19: 1617-1631, 1991.

[BLZ11] A. Batakis, P. Levitz, and M. Zinsmeister. Brownian flights. To appear in the $P A M Q, 2011$.

[Che93] Z-Q. Chen. On reflecting diffusions processes and skorohod decompositions. Probability theory and related fields, 94: 281-351, 1993.

[FOT94] M. Fukushima, Y. Oshima, and M. Takeda. Dirichlet Forms and Symmetric Markov Processes, volume 19 of Studies In Mathematics. de Gruyter, 1994. 
[Hör94] L. Hörmander. Notions of convexity. Birkhauser-Verlag Basel, 1994.

[Jon81] P. Jones. Quasiconformal mappings and extendability of functions in sobolev spaces. Acta Mathematica, 147 : 71-88, 1981.

[JW88] P. Jones and T. Wolff. Hausdorff dimension of harmonic measures in the plane. Acta Mathematica, 161 : 131-144, 1988.

[Mak85] N. G. Makarov. On the distortion of boundary sets under conformal mappings. Proceedings of the London Mathematical Society, 51 (3): 369-384, 1985. 